PDES, SUBMANIFOLDS AND

AFFINE DIFFERENTIAL GEOMETRY

BANACH CENTER PUBLICATIONS, VOLUME 69

INSTITUTE OF MATHEMATICS

POLISH ACADEMY OF SCIENCES

WARSZAWA 2005

\title{
TOPOLOGY AND GEOMETRY OF COMPLETE SUBMANIFOLDS IN EUCLIDEAN SPACES
}

\author{
QING-MING CHENG \\ Department of Mathematics \\ Faculty of Science and Engineering \\ Saga University, Saga 840-8502, Japan \\ E-mail: cheng@ms.saga-u.ac.jp
}

\begin{abstract}
This paper is a survey of results on topological structures and curvature structures
\end{abstract} of complete submanifolds in a Euclidean space.

1. Introduction. Let $M$ be an $n$-dimensional submanifold in an $n+p$-dimensional Euclidean space $\mathbb{E}^{n+p}$. We denote by $\mathfrak{M}(n)$ the set of $n$-dimensional submanifolds in Euclidean spaces. From a theorem of Nash, we know that every finite dimensional Riemannian manifold possesses an isometric embedding into a Euclidean space of sufficiently high dimension. Hence, $\mathfrak{M}(n)$ is too large. If we do not assume any condition, it is impossible to investigate the topology and geometry of such submanifolds. Therefore, we want to find a criterion under which we can study the topology and geometry of submanifolds in $\mathbb{E}^{n+p}$.

The paper is organized as follows. In section 2, we discuss the topology of complete submanifolds in $\mathbb{E}^{n+p}$. In section 3 , we consider the geometry of complete submanifolds with constant mean curvature in $\mathbb{E}^{n+p}$. In section 4 , we investigate the geometry of complete submanifolds with constant scalar curvature in $\mathbb{E}^{n+p}$. In section 5 , the geometry of complete hypersurfaces with constant mean curvature and constant scalar curvature in $\mathbb{E}^{n+1}$ is mentioned.

For the reader's convenience, we will review several basic facts on submanifolds. Let $M$ an $n$-dimensional connected submanifold in $\mathbb{E}^{n+p}$. We choose a local field of orthonormal frames $\left\{e_{1}, \ldots, e_{n+p}\right\}$ and the dual coframes $\left\{\omega_{1}, \ldots, \omega_{n+p}\right\}$ in such a way that, restricted

2000 Mathematics Subject Classification: Primary 53C42; Secondary 53C20.

Key words and phrases: submanifold, mean curvature, scalar curvature and second fundamental form.

The author's research was partially supported by a Grant-in-Aid for Scientific Research from the Japan Society for the Promotion of Science.

The paper is in final form and no version of it will be published elsewhere. 
to $M,\left\{e_{1}, \ldots, e_{n}\right\}$ are tangent to $M$. Let $\left\{\omega_{A B}\right\}$ denote the connection forms of $\mathbb{E}^{n+p}$. The canonical forms $\left\{\omega_{A}\right\}$ and connection forms $\left\{\omega_{A B}\right\}$ restricted to $M$ are also denoted by the same symbols. We then have

$$
\omega_{\alpha}=0, \quad \alpha=n+1, \ldots, n+p .
$$

We see that $e_{1}, \ldots, e_{n}$ is a local field of orthonormal frames adapted to the induced Riemannian metric on $M$ and $\omega_{1}, \ldots, \omega_{n}$ is a local field of its dual coframes on $M$. It follows from Cartan's Lemma that

$$
\omega_{\alpha i}=\sum_{j=1}^{n} h_{i j}^{\alpha} \omega_{j}, \quad h_{i j}^{\alpha}=h_{j i}^{\alpha} .
$$

The second fundamental form $\vec{\alpha}$ and the mean curvature vector $\mathbf{h}$ of $M$ are defined by

$$
\vec{\alpha}=\sum_{\alpha=n+1}^{n+p} \sum_{i, j=1}^{n} h_{i j}^{\alpha} \omega_{i} \omega_{j} e_{\alpha}, \quad \mathbf{h}=\frac{1}{n} \sum_{\alpha=n+1}^{n+p}\left(\sum_{i=1}^{n} h_{i i}^{\alpha}\right) e_{\alpha} .
$$

The mean curvature $H$ of $M$ is defined by

$$
H=\frac{1}{n} \sqrt{\sum_{\alpha=n+1}^{n+p}\left(\sum_{i=1}^{n} h_{i i}^{\alpha}\right)^{2}}
$$

Let $S=\sum_{\alpha=n+1}^{n+p} \sum_{i, j=1}^{n}\left(h_{i j}^{\alpha}\right)^{2}$ denote the squared norm of the second fundamental form of $M$. It is obvious that $S \geq n H^{2}$ holds. From the structure equations of $M$, we have the Gauss equation

$$
R_{i j k l}=\sum_{\alpha=n+1}^{n+p}\left(h_{i k}^{\alpha} h_{j l}^{\alpha}-h_{i l}^{\alpha} h_{j k}^{\alpha}\right)
$$

where $R_{i j k l}$ are the components of the curvature tensor of $M$. Letting $R_{i j}$ and $r$ denote components of the Ricci curvature and the scalar curvature of $M$, respectively, we have

$$
R_{j k}=\sum_{\alpha=n+1}^{n+p}\left(\sum_{i=1}^{n} h_{i i}^{\alpha} h_{j k}^{\alpha}-\sum_{i=1}^{n} h_{i k}^{\alpha} h_{j i}^{\alpha}\right), \quad r=n^{2} H^{2}-S .
$$

2. Topology of complete submanifolds. It is well known that the investigation of sphere theorems on Riemannian manifolds is very important in the study of differential geometry. It is our purpose in this section to consider differentiable sphere theorems of compact submanifolds in Euclidean spaces.

A classical theorem of Hadamard states that

THEOREM 2.1. An n-dimensional compact connected orientable hypersurface $M$ in $\mathbb{E}^{n+1}$ with positive sectional curvature is diffeomorphic to a standard sphere.

Let $N$ be Gauss map of $M$. We know that $N$ is a diffeomorphism from $M$ onto the unit sphere $S^{n}(1)$.

This above result of Hadamard was generalized by Van Heijenoort [38] and Sacksteder [35], who proved the following: 
THEOREM 2.2. An n-dimensional locally convex (that is, the second fundamental form is semi-definite) compact connected orientable hypersurface $M$ in $\mathbb{E}^{n+1}$ is diffeomorphic to a standard sphere.

REMARK 2.1. Since every finite dimensional Riemannian manifold possesses an isometric embedding into a Euclidean space of sufficiently high dimension, we cannot expect to extend these results of Hadamard, Van Heijenoort, and Sacksteder to higher codimensions because there exist many compact manifolds with positive sectional curvature, which are not diffeomorphic to a standard sphere. Hence, in order to obtain a differentiable sphere theorem on compact submanifolds in Euclidean spaces, the condition of positive sectional curvatures is not strong enough.

Chern and Lashof [13] and [14] studied the total curvature $c(M)$ of an $n$-dimensional compact connected orientable submanifolds in $\mathbb{E}^{n+p}$. The total curvature $c(M)$ is defined by

$$
c(M)=\int_{M} K^{*}(x) d M,
$$

where $K^{*}(x)=\int_{B_{\nu}(x)}|G(x, \nu)| d \sigma_{p-1}$ is the total curvature of $M$ at point $x, B_{\nu}(x)$ and $G(x, \nu)$ denote the bundle of unit normal vectors at $x$ and the Lipschitz-Killing curvature at $\nu(x)$, respectively. They proved

THEOREM 2.3. Let $M$ be an $n$-dimensional compact connected orientable submanifold in $\mathbb{E}^{n+p}$. Its total curvature satisfies the inequality

$$
c(M) \geq 2 c_{n+p-1}
$$

and if $c(M)=2 c_{n+p-1}$ holds, then $M$ is diffeomorphic to a standard sphere, where $c_{n+p-1}$ is the volume of the $n+p-1$-dimensional unit sphere $S^{n+p-1}(1)$.

Further, Shiohama and Xu [37] and Zhang [45] studied the topological sphere theorem of compact connected orientable submanifolds in $\mathbb{E}^{n+p}$. They proved

THEOREM 2.4. Let $M$ be an $n$-dimensional complete connected orientable submanifold in $\mathbb{E}^{n+p}$. If $\sup _{M}\left(S-\frac{n^{2} H^{2}}{n-1}\right)<0$, then $M$ is homeomorphic to a standard sphere when $n>3$, where $S$ and $H$ denote the squared norm of the second fundamental form and the mean curvature, respectively.

REMARK 2.2. From $\sup _{M}\left(S-\frac{n^{2} H^{2}}{n-1}\right)<0$, we know that the mean curvature $H \neq 0$ and it is also easy to prove the $\operatorname{Ricci}$ curvature $\operatorname{Ric}(M)$ is bounded from below by a positive constant (see [37] and [45]). From the Myers theorem, we have that $M$ is compact.

In order to prove Theorem 2.4, the following theorem of Lawson and Simons [27] and Xin [41] will be used:

THEOREM 2.5. Let $M$ be an $n$-dimensional compact submanifold in a space form $M^{n+p}(c)(c \geq 0)$. For $0<q<n$, any point $x \in M$ and any orthonormal frame $\left\{e_{i}\right\}$ in $T_{x} M$, if

$$
\sum_{i=1}^{q} \sum_{k=q+1}^{n} 2\left\|\vec{\alpha}\left(e_{i}, e_{k}\right)\right\|^{2}-\left\langle\vec{\alpha}\left(e_{i}, e_{i}\right), \vec{\alpha}\left(e_{k}, e_{k}\right)\right\rangle<q(n-q) c
$$


is satisfied, then $H_{q}(M, Z)=H_{n-q}(M, Z)=0$, where $\vec{\alpha}$ denotes the second fundamental form of $M$ and $H_{i}(M, Z)$ is the $i$-th homology group of $M$ with integer coefficients.

Proof of Theorem 2.4. (1) From $\sup _{M}\left(S-\frac{n^{2} H^{2}}{n-1}\right)<0$, we have that, for any $2 \leq q \leq n-2$,

$$
\sum_{i=1}^{q} \sum_{k=q+1}^{n} 2\left\|\vec{\alpha}\left(e_{i}, e_{k}\right)\right\|^{2}-\left\langle\vec{\alpha}\left(e_{i}, e_{i}\right), \vec{\alpha}\left(e_{k}, e_{k}\right)\right\rangle<q(n-q) c,
$$

is satisfied. From Theorem 2.5 of Lawson and Simons and Xin, we have $H_{2}(M, Z)=$ $H_{3}(M, Z)=\cdots=H_{n-2}(M, Z)=0$.

(2) From $\sup _{M}\left(S-\frac{n^{2} H^{2}}{n-1}\right)<0$, we have that $M$ is compact and the first fundamental group is finite. Hence, we obtain $H_{1}(M, Z)=H_{n-1}(M, Z)=0$.

(3) Let $\tilde{M}$ be universal covering of $M$. Then $\tilde{M}$ is homotopy sphere. From the Poincaré conjecture, $\tilde{M}$ is homeomorphic to a standard sphere. Hence, $M$ is homotopy sphere. By making use of the Poincaré conjecture again, we get that $M$ is homeomorphic to a standard sphere if $n>3$.

REMARK 2.3. From this proof, we can only infer that $M$ is homeomorphic to a standard sphere. We do not know whether $M$ is diffeomorphic to a standard sphere.

It is natural to ask under what condition $M$ is diffeomorphic to a standard sphere. In [7], Cheng proved:

THEOREM 2.6. An $n$-dimensional $(n \geq 3$ ) compact connected orientable submanifold $M$ with nonzero mean curvature $H$ in $\mathbb{E}^{n+p}$ is diffeomorphic to a standard sphere if $S \leq \frac{n^{2} H^{2}}{n-1}$ is satisfied and the normalized mean curvature vector is parallel, where $S$ denotes the squared norm of the second fundamental form of $M$.

REMARK 2.4. It is obvious that the condition $S \leq \frac{n^{2} H^{2}}{n-1}$ is weaker than $\sup _{M}\left(S-\frac{n^{2} H^{2}}{n-1}\right)<$ 0 and our result is stronger than the one of Shiohama and $\mathrm{Xu}$ and Zhang. In particular, when $M$ is a hypersurface, the condition that the normalized mean curvature vector is parallel is not necessary. Further, we need to remark that, in general, from the condition that the normalized mean curvature vector is parallel, we cannot obtain that the mean curvature is constant. Hence, it is weaker than the condition that the mean curvature vector is parallel. On the other hand, notice that in the main theorems 1 and 2 of Cheng [7], the condition that the normalized mean curvature vector is parallel was not assumed, although it is necessary. In the proof of Theorem 2.7, we shall explain why.

Next, we present a general result.

THEOREM 2.7. Let $M$ be an $n$-dimensional complete connected orientable submanifold with bounded nonzero mean curvature $H$ in $\mathbb{E}^{n+p}$. If the normalized mean curvature vector is parallel and the following inequality holds:

$$
S \leq \frac{n^{2} H^{2}}{n-1}
$$

then $M$ lies in a totally geodesic submanifold $\mathbb{E}^{n+1}$ of $\mathbb{E}^{n+p}$.

In order to prove Theorem 2.7, we need the following Generalized Maximum Principle of Omori [31] and Yau [44]: 
Theorem 2.8 (Generalized Maximum Principle, Omori [31] and Yau [44]). Let $M$ be an $n$-dimensional complete Riemannian manifold whose sectional curvature is bounded from below. If $F$ is a $C^{2}$-function bounded from above on $M$, then there exists a sequence $\left\{p_{m}\right\}$ of points in $M$ such that

$$
\lim _{m \rightarrow \infty} F\left(p_{m}\right)=\sup F, \quad \lim _{m \rightarrow \infty}\left|\operatorname{grad} F\left(p_{m}\right)\right|=0, \quad \lim _{m \rightarrow \infty} \sup \nabla_{k} \nabla_{k} F\left(p_{m}\right) \leq 0,
$$

for $k=1,2, \ldots, n$.

Proof of Theorem 2.7. Since the mean curvature of $M$ is not zero, we know that $e_{n+1}=\frac{\mathbf{h}}{H}$ is a normal vector field defined globally on $M$. We choose an orthonormal frame field

$$
\left\{e_{1}, \ldots, e_{n}, e_{n+1}, \ldots, e_{n+p}\right\}
$$

in $\mathbb{E}^{n+p}$ such that $\left\{e_{1}, \ldots, e_{n}\right\}$ are tangent to $M$. We define $S_{1}$ and $S_{2}$ by

$$
S_{1}:=\sum_{i, j=1}^{n}\left(h_{i j}^{n+1}-H \delta_{i j}\right)^{2}, \quad S_{2}:=\sum_{\alpha=n+2}^{n+p} \sum_{i, j=1}^{n}\left(h_{i j}^{\alpha}\right)^{2},
$$

respectively, where $h_{i j}^{\alpha}$ denote components of the second fundamental form of $M$. Then, $S_{1}$ and $S_{2}$ are functions defined on $M$ globally, which do not depend on the choice of the orthonormal frame $\left\{e_{1}, \ldots, e_{n}\right\}$. And

$$
S-n H^{2}=S_{1}+S_{2}
$$

Let $h_{i j k}^{\alpha}$ and $h_{i j k l}^{\alpha}$ denote components of the covariant differentiation and components of the second covariant differentiation of the second fundamental form of $M$, respectively. Then, we have (see [6] for details)

$$
\begin{aligned}
\frac{1}{2} \Delta S_{2}= & \sum_{\alpha=n+2}^{n+p} \sum_{i, j, k=1}^{n} h_{i j}^{\alpha} h_{k k i j}^{\alpha}+\sum_{\alpha=n+2}^{n+p} \sum_{i, j, k=1}^{n}\left(h_{i j k}^{\alpha}\right)^{2} \\
& +n H \sum_{\alpha=n+2}^{n+p} \operatorname{trace}\left(H_{n+1} H_{\alpha}^{2}\right)-\sum_{\alpha=n+2}^{n+p}\left[\operatorname{trace}\left(H_{n+1} H_{\alpha}\right)\right]^{2} \\
& -\sum_{\alpha, \beta=n+2}^{n+p} N\left(H_{\alpha} H_{\beta}-H_{\beta} H_{\alpha}\right)-\sum_{\alpha, \beta=n+2}^{n+p}\left[\operatorname{trace}\left(H_{\alpha} H_{\beta}\right)\right]^{2} \\
& +\sum_{\alpha=n+2}^{n+p} \operatorname{trace}\left(H_{n+1} H_{\alpha}\right)^{2}-\sum_{\alpha=n+2}^{n+p} \operatorname{trace}\left(H_{n+1}^{2} H_{\alpha}^{2}\right) .
\end{aligned}
$$

Since the normalized mean curvature $e_{n+1}$ is parallel, we have $\sum_{i} h_{i i k}^{\alpha}=0$ for any $\alpha \neq n+1$. Thus, we can infer $\sum_{\alpha=n+2}^{n+p} \sum_{i, j, k} h_{i j}^{\alpha} h_{k k i j}^{\alpha}=0$.

Here, we need to mention that in [6] and [7], $\sum_{\alpha=n+2}^{n+p} \sum_{i, j, k} h_{i j}^{\alpha} h_{k k i j}^{\alpha}=0$ was used without the assumption that the normalized mean curvature vector is parallel. But, in general, we cannot have this result if we do not have any assumption, which was pointed out by the referees of this paper. We would like to thank them for pointing out this mistake. 
From the calculations in [6], we can infer

$$
\frac{1}{2} \Delta S_{2} \geq \sum_{\alpha=n+2}^{n+p} \sum_{i, j, k=1}^{n}\left(h_{i j k}^{\alpha}\right)^{2}+\left\{\frac{(n-3)}{2} S_{2}\right\} S_{2} \geq 0 .
$$

Since the mean curvature is bounded, from the condition $S \leq \frac{n^{2} H^{2}}{n-1}$ and the Gauss equation, we know that the Ricci curvature of $M$ is bounded from below. By applying Theorem 2.8 to the function $S_{2}$, we have that there exists a sequence $\left\{p_{k}\right\} \subset M$ such that

$$
\lim _{k \rightarrow \infty} S_{2}\left(p_{k}\right)=\sup S_{2}, \quad \lim _{k \rightarrow \infty} \sup \Delta S_{2}\left(p_{k}\right) \leq 0 .
$$

Hence, $\sup S_{2}=0$, that is, $S_{2}=0$ on $M$. Thus, we have

$$
\sum_{\alpha=n+2}^{n+p} \sum_{i, j, k=1}^{n}\left(h_{i j k}^{\alpha}\right)^{2}=0
$$

on $M$.

Since $e_{n+1}$ is parallel in the normal bundle $T^{\perp}(M)$ of $M$, if we denote by $N_{1}$ the normal subbundle spanned by $e_{n+2}, e_{n+3}, \ldots, e_{n+p}$ of the normal bundle of $M$, then $M$ is totally geodesic with respect to $N_{1}$. Since $e_{n+1}$ is parallel in the normal bundle, we know that the normal subbundle $N_{1}$ is invariant under parallel translation with respect to the normal connection of $M$. Then from Theorem 1 in [37], we conclude that $M$ lies in a totally geodesic submanifold $\mathbb{E}^{n+1}$ of $\mathbb{E}^{n+p}$. This finishes our proof.

Now, we can deduce our Theorem 2.6 from Theorem 2.7.

Proof of Theorem 2.6. Since $M$ is compact, we know that the mean curvature is bounded. From the condition $S \leq \frac{n^{2} H^{2}}{n-1}$, we know that Theorem 2.7 is true. Therefore, $M$ lies in a totally geodesic submanifold $\mathbb{E}^{n+1}$ of $\mathbb{E}^{n+p}$. We denote by $H^{\prime}$ the mean curvature of $M$ in $\mathbb{E}^{n+1}$. Since $\mathbb{E}^{n+1}$ is totally geodesic in $\mathbb{E}^{n+p}$, we have $H=H^{\prime}$, that is, the mean curvature $H^{\prime}$ of $M$ in $\mathbb{E}^{n+1}$ is the same as in $\mathbb{E}^{n+p}$. We can also obtain that the squared norm $S^{\prime}$ of the second fundamental form of $M$ in $\mathbb{E}^{n+1}$ is the same as in $\mathbb{E}^{n+p}$. Thus, we can conclude $S^{\prime} \leq \frac{n^{2}\left(H^{\prime}\right)^{2}}{n-1}$ and $H^{\prime} \neq 0$. From this condition and the Gauss equation, we can prove that the sectional curvatures of $M$ are nonnegative (see p. 483 in [7] in details). Since $H^{\prime} \neq 0$, we can prove that $M$ is a locally convex hypersurface. From the result of Van Heijenoort and Sacksteder, we infer that $M$ is diffeomorphic to a standard sphere.

3. Geometry of complete submanifolds with constant mean curvature. In this section, we shall study the geometry of complete connected orientable submanifolds with constant mean curvature in $\mathbb{E}^{n+p}$.

In 1900, Liebmann proved that a strictly convex, compact surface of constant mean curvature in $\mathbb{E}^{3}$ is isometric to a standard sphere. As a generalization of the above result, in 1951, Hopf [20] proved that the only possible differentiable immersions of a sphere into $\mathbb{E}^{3}$ with constant mean curvature are exactly those standard spheres.

Further, he proposed the following conjecture:

Conjecture 3.1 (Hopf's conjecture). An n-dimensional compact connected orientable hypersurface with constant mean curvature in $\mathbb{E}^{n+1}$ is isometric to a standard sphere. 
For Hopf's conjecture, Alexandrov [2] obtained an important contribution. He proved the following:

THEOREM 3.1. If $M$ is an $n$-dimensional embedded compact connected orientable hypersurface in $\mathbb{E}^{n+1}$ with constant mean curvature, then $M$ is isometric to a standard sphere.

Next, we shall give a proof of Theorem 3.1 of Alexandrov by making use of the so-called Reilly formula in [33].

REILlY'S FORMUlA. Let $\Omega$ be a bounded domain in $\mathbb{E}^{n+1}$ with boundary $M=\partial \Omega$. Given a function $f \in C^{\infty}(\bar{\Omega})$, we put $g=f_{\mid M}$ and $u=\partial f / \partial N$. Then

$$
\int_{\Omega}\left\{(\bar{\Delta} f)^{2}-|\operatorname{Hess} f|^{2}\right\} d v=\int_{M}\left\{-2(\Delta g) u+n H u^{2}+\vec{\alpha}(\operatorname{grad} g, \operatorname{grad} g)\right\} d M,
$$

where $\bar{\Delta} f$ and Hess $f$ denote the Laplacian and the Hessian of $f$ in $\mathbb{E}^{n+1}$, respectively, and $N$ denotes the inner normal vector field on $M=\partial \Omega$.

Proof of Theorem 3.1. (1) Since $M$ is an embedding, we know that there exists a domain $\Omega \subset \mathbb{E}^{n+1}$ such that $M=\partial \Omega$.

(2) From Reilly's formula, by taking $\bar{\Delta} f=1$ in $\Omega$ and $g=f_{\mid M}=0$, we have

$$
\int_{M} \frac{1}{H} d M \geq(n+1) \operatorname{vol} \Omega
$$

and the equality holds if and only if $M$ is isometric to a sphere.

(3) Let $\phi$ denote the position vector field of $\mathbb{E}^{n+1}$ with respect to the origin. From the so-called Minkowski formula (see [22] and [23])

$$
\int_{M}(1+H\langle\phi, N\rangle) d M=0
$$

and

$$
-\int_{M}\langle\phi, N\rangle d M=(n+1) \operatorname{vol} \Omega,
$$

we obtain

$$
\int_{M} \frac{1}{H} d M=(n+1) \operatorname{vol} \Omega
$$

(4) From (2) and (3), we have that $M$ is isometric to a standard sphere.

The basic problem whether there exist any other oriented compact immersed hypersurfaces in $\mathbb{E}^{n+1}$ with constant mean curvature was open until W. Y. Hsiang [21] and Wente [39] discovered examples which are not isometric to a standard sphere. In both cases, there exist infinitely many examples of compact immersed hypersurfaces in $\mathbb{E}^{n+1}$ $(n \geq 2)$ with constant mean curvature, which are not isometric to a standard sphere. Of course, they are not embeddings.

Wente's examples

(1) They are compact surfaces with constant mean curvature in $\mathbb{E}^{3}$.

(2) They have genus $=1$.

Furthermore, Kapouleas [20] constructed new examples of compact surfaces immersed into $\mathbb{E}^{3}$ with constant mean curvature and higher genus. 
Hsiang's examples

(1) They are compact hypersurfaces with constant mean curvature in $\mathbb{E}^{n+1}(n \geq 3)$.

(2) They are not isometric to a standard sphere.

(3) They are diffeomorphic to a standard sphere.

From the examples of Hsiang, we know that although they are not isometric to a standard sphere, they are diffeomorphic to a sphere. That is, in the topological sense, they are a standard sphere. Hence, we can ask the following:

Problem 3.1. Is it true that an n-dimensional compact connected orientable hypersurface with constant mean curvature in $\mathbb{E}^{n+1}(n \geq 3)$ is diffeomorphic to a standard sphere?

Next, we consider complete connected orientable submanifolds in $\mathbb{E}^{n+p}$.

It is well known that Klotz and Osserman [25] proved the following:

THEOREM 3.2. A complete connected orientable surface $M^{2}$ with constant mean curvature $H$ in $\mathbb{E}^{3}$ is isometric to a totally umbilical sphere $S^{2}(c)$, a totally geodesic plane $\mathbb{E}^{2}$ or a cylinder $\mathbb{E}^{1} \times S^{1}(c)$ if its Gaussian curvature is nonnegative.

REMARK 3.1. It is well known that the Gaussian curvature is nonnegative if and only if $S \leq \frac{n^{2} H^{2}}{n-1}$ holds in the case of $n=2$, where $S$ denotes the squared norm of the second fundamental form.

It is a natural problem to generalize the result of Klotz and Osserman to higher dimensions and higher codimensions. In [11], S.Y. Cheng and Yau proved

THEOREM 3.3. Let $M$ be an $n$-dimensional complete connected orientable hypersurface with constant mean curvature in $\mathbb{E}^{n+1}$. If the sectional curvature of $M$ is nonnegative, then $M$ is isometric to a standard sphere, a hyperplane $\mathbb{E}^{n}$ or a Riemannian product $\mathbb{E}^{k} \times S^{n-k}(c), 1 \leq k \leq n-1$.

Proof. (1) When $M$ is compact, from

$$
\frac{1}{2} \Delta S=\sum_{i, j, k=1}^{n} h_{i j k}^{2}+\sum_{i<j=1}^{n}\left(\lambda_{i}-\lambda_{j}\right)^{2} K_{i j} .
$$

and Stokes formula, we know that $M$ is isometric to a standard sphere.

Next, we assume that $M$ is complete and noncompact.

(2) Since the sectional curvatures of $M$ are nonnegative, there exists a unit vector $\vec{a}$ in $\mathbb{E}^{n+1}$ such that $\langle N, \vec{a}\rangle \geq 0$.

(3) If $H=0$, then $M$ is isometric to a hyperplane because the sectional curvatures of $M$ are nonnegative.

(4) In the case $H \neq 0$, since the sectional curvatures of $M$ are nonnegative, we can prove that the lower bound $\lambda_{1}(M)$ of the spectrum of Laplacian on $M$ satisfies

$$
0=\lambda_{1}(M) \geq \inf (-\Delta f / f)
$$

for any positive smooth function $f$. From $\Delta\langle N, \vec{a}\rangle=-S\langle N, \vec{a}\rangle$ and the maximum principle, we have $\langle N, \vec{a}\rangle=0$.

(5) From $\langle N, \vec{a}\rangle=0$, we infer that $M$ is isometric to a Riemannian product $\mathbb{E}^{k} \times$ $S^{n-k}(c), 1 \leq k \leq n-1$. 
In [8], Cheng and Nonaka proved

THEOREM 3.4. Let $M$ be an $n$-dimensional $(n>2)$ complete connected orientable submanifold with parallel mean curvature vector in $\mathbb{E}^{n+p}$. If $S \leq \frac{n^{2} H^{2}}{n-1}$ is satisfied, then $M$ is isometric to a totally umbilical sphere $S^{n}(c)$, a totally geodesic hyperplane $\mathbb{E}^{n}$ or a generalized cylinder $\mathbb{E}^{1} \times S^{n-1}(c)$, where $S$ and $H$ denote the squared norm of the second fundamental form and the mean curvature of $M$.

Remark 3.2. In 1985, Y. B. Shen [36] tried to prove Theorem 3.4. But his proof is not correct because he used a wrong result of Motomiya [29] which plays an essential role in his proof. In fact, Motomiya [29] wanted to extend the generalized maximum principle of Yau [44]. But his result is wrong. The counterexamples were constructed in [10] (see [8] and [10] for details).

Proof of Theorem 3.4. From $S \leq \frac{n^{2} H^{2}}{n-1}$ and $H=$ constant, we know that $M$ is isometric to a hyperplane if $H=0$. When $H \neq 0$, from Theorem 2.7 and the proof of Theorem 2.6, we know that $M$ is a complete hypersurface with nonnegative sectional curvatures. From Theorem 3.3 of S. Y. Cheng and Yau, we know that Theorem 3.4 is true.

4. Geometry of complete submanifolds with constant scalar curvature. In this section, we shall discuss complete connected orientable submanifolds with constant scalar curvature in $\mathbb{E}^{n+p}$. It is well known that in 1982, Yau proposed the following conjecture in [42]:

Conjecture 4.1 (Yau's conjecture). An n-dimensional compact connected orientable hypersurface with constant scalar curvature in $\mathbb{E}^{n+1}$ is isometric to a standard sphere.

We should compare Yau's conjecture with Hopf's conjecture. Although Hopf's conjecture was solved negatively, Yau's conjecture is still open.

For Hopf's conjecture, Alexandrov proved that Hopf's conjecture is true if the hypersurface is an embedding. Concerning Yau's conjecture, by making use of Reilly's formula, Ros [34] also gave an affirmative answer if the hypersurface is an embedding.

THEOREM 4.1. If $M$ is an $n$-dimensional embedded compact connected orientable hypersurface in $\mathbb{E}^{n+1}$ with constant scalar curvature, then $M$ is isometric to a standard sphere.

Proof. (1) Since $M$ is an embedding, we know that there exists a domain $\Omega \subset \mathbb{E}^{n+1}$ such that $M=\partial \Omega$.

(2) Since $M$ is compact and the scalar curvature is constant, we have $H \neq 0$. We can assume $H>0$ on $M$. From the Gauss equation and $S \geq n H^{2}$, we have $r \leq n(n-1) H^{2}$ and equality holds if and only if $M$ is isometric to a standard sphere.

(3) Let $\phi$ denote the position vector field of $\mathbb{E}^{n+1}$ with respect to the origin. From Minkowski's formula

$$
\int_{M}(n(n-1) H+r\langle\phi, N\rangle) d M=0
$$

we have

$$
\int_{M} H d M=\frac{n+1}{n(n-1)} r \operatorname{vol} \Omega
$$


(4) From (2) and (3), we can infer

$$
\sqrt{n(n-1)} \operatorname{vol} M \leq(n+1) \sqrt{r} \operatorname{vol} \Omega
$$

and equality holds if and only if $M$ is isometric to a standard sphere.

(5) Let $f$ satisfy $\bar{\Delta} f=1$ in $\Omega$ and $f=0$ on $\partial \Omega$. From Reilly's formula, we have

$$
\frac{\operatorname{vol} \Omega}{n+1} \geq \int_{M} H u^{2} d M,
$$

where $u=\partial f / \partial N$.

(6) From Schwarz inequality and (5), we can obtain

$$
\sqrt{n(n-1)} \operatorname{vol} M \geq(n+1) \sqrt{r} \operatorname{vol} \Omega .
$$

(7) From (4) and (6), we infer that $M$ is isometric to a standard sphere.

When we do not assume that $M$ is an embedding, Cheng [5] proved

THEOREM 4.2. An n-dimensional $(n>3)$ compact connected orientable locally conformally flat hypersurface with constant scalar curvature in $\mathbb{E}^{n+1}$ is isometric to a standard sphere.

Proof. (1) From a result of Cartan and Schouten (see [16]), we have that $M$ has at most two distinct principal curvatures.

(2) We can prove that there exist no compact locally conformally flat hypersurfaces with constant scalar curvature and with two distinct principal curvatures.

(3) If $M$ has two distinct principal curvatures at some point, then $M$ has two distinct principal curvatures on $M$.

(4) From (2) and (3), we conclude that $M$ is isometric to a standard sphere.

Next, we consider complete connected orientable submanifolds in $\mathbb{E}^{n+p}$.

By a classical result and a theorem of Hartman and Nirenberg [18], we know that a complete connected orientable surface with constant curvature in $\mathbb{E}^{3}$ is isometric to a totally umbilical sphere $S^{2}(c)$, a totally geodesic plane $\mathbb{E}^{2}$ or a cylinder $\mathbb{E}^{1} \times S^{1}(c)$.

In this case, we know that the Gaussian curvature is nonnegative. As a generalization of this result, S. Y. Cheng and Yau [10] proved

THEOREM 4.3. Let $M$ be an $n$-dimensional complete connected orientable hypersurface with constant scalar curvature in $\mathbb{E}^{n+1}$. If the sectional curvature of $M$ is nonnegative, then $M$ is isometric to a standard sphere, a hyperplane $\mathbb{E}^{n}$ or a Riemannian product $\mathbb{E}^{k} \times S^{n-k}(c), 1 \leq k \leq n-1$.

Proof. (1) When $M$ is compact, a differential operator $\square$ acting on functions $f$ is defined by

$$
\square f=\sum_{i, j=1}^{n}\left(n H \delta_{i j}-h_{i j}^{n+1}\right) f_{i j},
$$


where $d f=\sum_{i=1}^{n} f_{i} \omega_{i}, \sum_{i j=1}^{n} f_{i j} \omega_{j}=d f_{i}+\sum_{j=1}^{n} f_{j} \omega_{j i}$. Since the scalar curvature is constant, we have

$$
\square(n H)=\sum_{i, j, k=1}^{n} h_{i j k}^{2}-|\operatorname{grad}(n H)|^{2}+\sum_{i<j=1}^{n}\left(\lambda_{i}-\lambda_{j}\right)^{2} K_{i j} .
$$

From this formula and Stokes formula, we know that $M$ is isometric to a standard sphere.

Next, we assume that $M$ is complete and noncompact.

(2) Since the sectional curvatures of $M$ are nonnegative, there exists a unit vector $\vec{a}$ in $\mathbb{E}^{n+1}$ such that $\langle N, \vec{a}\rangle \geq 0$.

(3) If the scalar curvature is zero, then $M$ is flat because the sectional curvatures of $M$ are nonnegative. From a result of Hartman and Nirenberg [18], the assertion is true.

(4) In the case $r>0$, since the sectional curvatures of $M$ are nonnegative, by making use of

$$
\square\langle N, \vec{a}\rangle=-\sum_{k, l}\left(n H \delta_{k l}-h_{k l}\right) \sum_{i} h_{k i} h_{i l}\langle N, \vec{a}\rangle,
$$

we know that $\langle N, \vec{a}\rangle=0$.

(5) From $\langle N, \vec{a}\rangle=0$, we infer that $M$ is isometric to a Riemannian product $\mathbb{E}^{k} \times$ $S^{n-k}(c), 1 \leq k \leq n-1$.

In [6] Cheng proved

THEOREM 4.4. Let $M$ be an $n$-dimensional $(n>2)$ complete connected orientable submanifold with constant scalar curvature $r$ in $\mathbb{E}^{n+p}$. Assume that at points where the mean curvature is not zero, the normalized mean curvature vector is parallel. If $S \leq \frac{r}{n-2}$ is satisfied, then $M$ is isometric to a totally umbilical sphere $S^{n}(c)$, a totally geodesic hyperplane $\mathbb{E}^{n}$ or a generalized cylinder $\mathbb{E}^{1} \times S^{n-1}(c)$, where $S$ denotes the squared norm of the second fundamental form of $M$.

REMARK 4.1. Notice that in Theorems 1.1 and 1.3 of Cheng [6], the condition that the normalized mean curvature vector is parallel is not assumed. As we remarked in Remark 2.4 , this condition is necessary.

Proof of Theorem 4.4. For any $n \geq 3$, from the Gauss equation, we know $r=n^{2} H^{2}-S$. Hence, $S \leq \frac{r}{n-2}$ holds if and only if $S \leq \frac{n^{2} H^{2}}{n-1}$ is satisfied. If $H=0$ holds at some point, we have $r=0$ on $M$. Hence, $M$ is flat. From a result of Hartman and Nirenberg [18], we know that Theorem 4.4 is true. When $H \neq 0$, from Theorem 2.7 and the proof of Theorem 2.6, we know that $M$ is a complete hypersurface with nonnegative sectional curvatures. From Theorem 4.3, we know that Theorem 4.4 is true.

5. Geometry of complete hypersurfaces. In this section, we only consider hypersurfaces with constant mean curvature and constant scalar curvature in $\mathbb{E}^{n+1}$. It is well known that an $n$-dimensional compact hypersurface $M$ in $\mathbb{E}^{n+1}$ with constant mean curvature and constant scalar curvature is isometric to a standard sphere (see [28] for finding a proof).

On the other hand, for complete and noncompact hypersurfaces, it is well known that the Riemannian products $\mathbb{E}^{k} \times S^{n-k}(c)$ for $k=1,2, \ldots, n-1$ are $n$-dimensional 
complete hypersurfaces with constant mean curvature and constant scalar curvature in $\mathbb{E}^{n+1}$, which are called generalized cylinders. From Theorem 5.1, it is natural to ask the following:

Problem 5.1. Let $M$ be an n-dimensional complete hypersurface in $\mathbb{E}^{n+1}$ with constant mean curvature and constant scalar curvature. Is it true that then $M$ is isometric to a standard sphere, a hyperplane $\mathbb{E}^{n}$ or a Riemannian product $\mathbb{E}^{k} \times S^{n-k}(c)$ for $k=$ $1,2, \ldots, n-1$ ?

REMARK 5.1. When $n=2$, it is obvious that the statement in problem 5.1 holds. When $n=3$, the author and Wan [9] gave an affirmative answer for it (see Theorem 5.1). For $n>3$, it is still open.

TheOREM 5.1. Let $M$ be a 3-dimensional complete hypersurface in $\mathbb{E}^{4}$ with constant mean curvature. If the scalar curvature is constant, then $M$ is isometric to a standard sphere, a hyperplane $\mathbb{E}^{3}$ or a Riemannian product $\mathbb{E}^{k} \times S^{3-k}(c)$ for $k=1,2$.

Proof. (1) Since $M$ is a hypersurface with constant mean curvature and with constant scalar curvature, we have the following:

$$
\sum_{i, j, k} h_{i j k}^{2}=S^{2}-3 H f_{3},
$$

where $f_{3}=\sum_{i} \lambda_{i}^{3}$, and

$$
\begin{aligned}
& \sum_{i, j, k, l} h_{i j k l}^{2}=-\frac{5}{3} \sum_{i, j, k}\left(\mu_{i}+\mu_{j}+\mu_{k}\right)^{2} h_{i j k}^{2}-24 H \sum \mu_{i} h_{i j k}^{2} \\
& +\frac{3}{2}\left(S-6 H^{2}\right) \sum h_{i j k}^{2}+\frac{3}{2}\left(S-3 H^{2}\right)\left(S-\frac{9}{2} H^{2}\right)\left(S-9 H^{2}\right),
\end{aligned}
$$

where $\mu_{i}=\lambda_{i}-H$.

(2) If there exists a point $x \in M$ such that, at $x, M$ has at most two distinct principal curvatures, then by estimating $\sum h_{i j k l}^{2}$, we conclude that $M$ is an isoparametric hypersurface with at most two distinct principal curvatures.

(3) If $M$ has three distinct principal curvatures at every point in $M$, by making use of the formulas in (1) and applying the Generalized Maximum Principle of Omori [31] and Yau [44] to the function $f_{3}=\sum_{i=1}^{3}\left(\lambda_{i}\right)^{3}$, we infer that this is impossible.

(4) From a result of Cartan [3], we conclude that Theorem 5.1 is true.

Acknowledgements. We like to express our gratitude to the referees for pointing out a mistake in our paper [6] and for other valuable suggestions and comments.

\section{References}

[1] U. Abresch, Constant mean curvature tori in terms of elliptic functions, J. Reine Angew. Math. 374 (1987), 169-192.

[2] A. D. Alexandrov, Uniqueness theorems for surfaces in the large, Vestnik Leningrad Univ. 13 (1958), 5-8.

[3] E. Cartan, Sur des familles remarquables d'hypersurfaces isoparamétriques dans les espaces sphériques, Math. Z. 45 (1939), 335-367. 
[4] B. Y. Chen, Geometry of Submanifolds, Marcel Dekker, New York, 1973.

[5] Q. M. Cheng, Complete hypersurfaces in a Euclidean space $\mathbf{R}^{n+1}$ with constant scalar curvature, Indiana Univ. Math. J. 51 (2002), 53-68.

[6] Q. M. Cheng, Submanifolds with constant scalar curvature, Proc. Royal Society Edinburgh 132 A (2002), 1163-1183.

[7] Q. M. Cheng, Spherical rigidities of submanifolds in Euclidean spaces, J. Math. Soc. Japan 56 (2004), 475-487.

[8] Q. M. Cheng and K. Nonaka, Complete submanifolds in Euclidean spaces with parallel mean curvature vector, Manuscripta Math. 105 (2001), 353-366.

[9] Q. M. Cheng and Q. R. Wan, Complete hypersurfaces of $\mathbf{R}^{4}$ with constant mean curvature, Mh. Math. 118 (1994), 171-204.

[10] Q. M. Cheng and B. Q. Wu, The generalized maximum principle and conformally flat spaces, Northeastern Math. J. 8 (1992), 54-56.

[11] S. Y. Cheng and S. T. Yau, Differential equations on Riemannian manifolds and their geometric applications, Comm. Pure Appl. Math. 28 (1975), 333-354.

[12] S. Y. Cheng and S. T. Yau, Hypersurfaces with constant scalar curvature, Math. Ann. 225 (1977), 195-204.

[13] S. S. Chern and R. K. Lashof, On the total curvature of immersed manifolds, Amer. J. Math. 79 (1957), 306-318.

[14] S. S. Chern and R. K. Lashof, On the total curvature of immersed manifolds II, Michigan Math. J. 5 (1958), 5-12.

[15] G. Darboux, Leçons sur la théorie générale des surfaces, Gauthier-Villars, Paris, 1896.

[16] M. do Carmo, M. Dajczer and F. Mercuri, Compact conformally flat hypersurfaces, Trans. Amer. Math. Soc. 288 (1985), 189-203.

[17] J. A. Erbacher, Reduction of the codimension of an isometric immersion, J. Differential Geom. 5 (1971), 333-340.

[18] P. Hartman and L. Nirenberg, On spherical image maps whose Jacobians do not change sign, Amer. J. Math. 81 (1959), 901-920.

[19] E. Heintze and H. Karcher, A general comparison theorem with applications to volume estimates for submanifolds, Ann. Sci. Ecole Norm. Sup. 11 (1978), 451-470.

[20] H. Hopf, Differential Geometry in the Large, Lecture Notes in Math. 1000, SpringerVerlag, 1983.

[21] W. Y. Hsiang, Generalized rotational hypersurfaces of constant mean curvature in the Euclidean spaces I, J. Differential Geom. 17 (1982), 337-356.

[22] C. C. Hsiung, Some integral formulas for closed hypersurfaces, Math. Scand. 2 (1954), 286-294.

[23] C. C. Hsiung, Some integral formulas for closed hypersurfaces in Riemannian space, Pacific J. Math. 6 (1956), 291-299.

[24] N. Kapouleas, Constant mean curvature surfaces in Euclidean three-space, Bull. Amer. Math. Soc. 17 (1987), 318-320.

[25] T. Klotz and R. Osserman, On complete surfaces in $\mathbf{E}^{3}$ with constant mean curvature, Comment. Math. Helv. 41 (1966-67), 313-318.

[26] N. J. Korevaar, Sphere theorems via Alexandrov for constant Weingarten curvature hypersurfaces-Appendix to a note of A. Ros, J. Differential Geom. 27 (1988), 221-223.

[27] H. B. Lawson and J. Simons, On stable currents and their application to global problems in real and complex geometry, Ann. of Math. 98 (1973), 427-450. 
[28] S. Montiel and A. Ros, Compact hypersurfaces: The Alexandrov theorem for higher order mean curvatures, in: Differential Geometry, H. B. Lawson and B. Tenenblat (eds.), New York, 1991, 279-296.

[29] K. Motomiya, On functions which satisfy some differential inequalities on Riemannian manifolds, Nagoya Math. J. 81 (1981), 57-72.

[30] K. Nomizu and B. Smyth, A formula of Simons' type and hypersurfaces with constant mean curvature, J. Differential Geom. 3 (1969), 367-377.

[31] H. Omori, Isometric immersions of Riemannian manifolds, J. Math. Soc. Japan 19 (1967), 205-214.

[32] U. Pinkall, Compact conformally flat hypersurfaces, in: Conformal Geometry, R. S. Kulkarni and U. Pinkall (eds.), Aspects Math. E12, Max-Planck-Inst. Math., 1988, 217-236.

[33] R. Reilly, Applications of the Hessian operator in a Riemannian manifold, Indiana Univ. Math. J. 26 (1977), 459-472.

[34] A. Ros, Compact hypersurfaces with constant scalar curvature and a congruence theorem, J. Differential Geom. 27 (1988), 215-220.

[35] R. Sacksteder, On hypersurfaces with no negative sectional curvature, Amer. J. Math. 82 (1960), 609-630.

[36] Y. B. Shen, Complete submanifolds in $\mathbb{E}^{n+p}$ with parallel mean curvature, Chin. Ann. of Math. Ser. B 6 (1985), 345-350.

[37] K. Shiohama and H. W. Xu The topological sphere theorem for complete submanifolds, Compositio Math. 107 (1997), 221-232.

[38] J. Van Heijenoort, On locally convex manifolds, Comm. Pure Appl. Math. 5 (1952), 223-242.

[39] H. C. Wente, Counterexample to a conjecture of H. Hopf, Pacific J. Math. 121 (1986), 193-243.

[40] H. Wu, The spherical images of convex hypersurfaces, J. Differential Geom. 9 (1974), 297-290.

[41] Y. L. Xin, Application of integral currents to the vanishing theorems, Scient. Sinica (A), 27 (1984), 233-241.

[42] S. T. Yau (ed.), Seminar on Differential Geometry, Annals of Math. Studies 102, Princeton Univ. Press, 1982.

[43] S. T. Yau, Submanifolds with constant mean curvature I, Amer. J. Math. 96 (1974), 346-366.

[44] S. T. Yau, Harmonic functions on complete Riemannian manifolds, Comm. Pure and Appl. Math. 28 (1975), 201-228.

[45] X. S. Zhang, Geometry and topology of submanifolds immersed in space forms and ellipsoids, Kodai Math. J. 17 (1994), 262-272. 